\title{
Hallazgos histológicos de biopsias obtenidas por endoscopia digestiva superior en el paciente pediátrico
}

\author{
Histological findings of biopsies obtained by upper gastrointestinal tract endoscopy in children
} Carlos Felipe Ponce, * Dilma Celenia Godoy, * Ruth Maricela Banegas**

\section{RESUMEN}

Antecedentes: El desarrollo de la endoscopia ha dado pasos agigantados en el campo de la gastroenterología pediátrica, convirtiéndose en una prueba diagnóstica de primera línea por la posibilidad de toma de biopsia, además se ha consolidado como un tratamiento eficaz en numerosas enfermedades digestivas. Objetivo: revisar los hallazgos histológicos de biopsias obtenidas por endoscopia digestiva superior en el paciente pediátrico del Hospital Nacional Dr. Mario Catarino Rivas (HNMCR) en el período comprendido entre enero a mayo 2016. Pacientes y métodos: Estudio transversal, descriptivo, realizado en pacientes con edades comprendidas entre 28 días y 18 años. Se revisaron 40 biopsias que cumplían los criterios de inclusión. Los datos se obtuvieron de los archivos de biopsias del servicio de patología, posteriormente se hizo un análisis estadístico de las variables. Resultados: se revisaron 40 biopsias, 55\% hombres, $45 \%$ mujeres, el $47.5 \%$ de los pacientes eran adolescentes. La indicación mas común fue el dolor abdominal crónico (33\%), seguido por la gastritis crónica (28\%) y reflujo gastroesofágico (20\%). Los hallazgo histopatológico más frecuente fueron la gastritis crónica $43 \%$, esofagitis crónica $30 \%$ y el Helycobacter Pylori 13\%. Conclusión: En el 95\% de las biopsias realizadas se encontraron anormalidades, siendo el más frecuente la gastritis crónica.

\section{PALABRAS CLAVE}

Endoscopía, gastritis crónica, hallazgos histológicos.

* Residente de 3er año de Pediatria, Universidad Nacional Autónoma de Honduras en el Valle de

Sula (UNAH-VS)

** Gastroenteróloga Pediatra, HNMCR

Dirigir correspondencia a: cponce_xs@hotmail.com

Recibido: 17 dejunio 2016 Aprobado 27 de agosto 2016

\section{SUMMARY}

Background: The development of endoscopy has made huge step in the field of pediatric gastroenterology, becoming a first line diagnostic test by the possibility of taking biopsy, beside it has established itself as an effective treatment in a number of digestive diseases. Objective: To review the histological findings of biopsies obtained by upper endoscopy, at Children's Hospital Patient Dr. Mario Catarino Rivas (HNMCR) In a period from January 1 May 2014. Patients and Methods: Retrospective, Descriptive, Made Study patients aged between 28 days and 18 years. 40 biopsies that met the inclusion criteria were reviewed. Data were obtained from the Archives of Pathology Service biopsies later became an statistical analysis of the variables. Results: 40 biopsies, $55 \%$ male, $45 \%$ female, $47.5 \%$ of patients were adolescents were reviewed. The most common indication was chronic abdominal pain (33\%), followed by chronic gastritis (28\%) and gastroesophagic reflux (20\%). The histopathologic more Frequent were Chronic gastritis 43\%, 30\% chronic esophagitis and Helicobacter pylori $13 \%$. Conclusion: In $95 \%$ of realized biopsies abnormalities the most frequent abnormalites finding was chronic gastritis.

\section{KEYWORDS:}

Endoscopy, chronic gastritis, histological findings.

\section{INTRODUCCIÓN}

El deseo humano para mirar dentro del cuerpo fue la fuerza impulsora en el desarrollo de la endoscopia. El campo de gastroenterología comenzó con Philip Bozzini's y Lichtleiter en 1805 y floreció con la introducción de la endoscopia gastrointestinal flexible por Basil Hirschowatz a finales del 1950, desde los años 
70 la endoscopia ha sido una herramienta diagnóstica, terapéutica y y de seguimiento en el paciente pediátrico, gracias a esta técnica es posible el estudio de las lesiones mucosas superficiales y de la hemorragia gastrointestinal permitiéndonos detallar su historia natural a través de la observación in vivo y además la toma de biopsia para el estudio histopatológico que potencia su valor diagnóstico. ${ }^{(1,2)}$

En pediatría, el dolor abdominal es una indicación común para realizar endoscopia. Por ejemplo, los datos de PEDS-CORI (Pediatric Endoscopy Database System-Clinical Outcomes Research Initiative- "Sistema de Base de Datos de Endoscopía Pediátrica - Iniciativa de Investigación de Resultados Clínicos") revelan que entre 17,180 Endoscopias realizadas, la indicación más frecuente fue el dolor abdominal $6,528(38 \%)$ de los casos. ${ }^{(3,4)}$

En la patología pediátrica las indicaciones de la biopsia endoscópica se han expandido en los últimos años, lo que ha contribuido al diagnóstico temprano y tratamiento adecuado de diversas patologías como la constipación crónica del lactante, la diarrea del niño inmunosuprimido, la enfermedad inflamatoria intestinal, etc. ${ }^{(5)}$

Un reciente informe técnico de la Academia Americana de Pediatría y NASPGHAN (Sociedad Norteamericana de Gastroenterología, Hepatología y Nutrición) llegó a la conclusión de que, en la evaluación del dolor abdominal crónico "hay poca evidencia para sugerir el uso de la endoscopia y biopsia en ausencia de síntomas de alarma".(6-8)

En Latinoamérica la Asociación Colombiana de Gastroenterología en el año 2014 publicó un estudio cuyo objetivo fue definir el valor diagnóstico de la endoscopia en pacientes menores de 18 años encontrando que en un período de 20 años dichos procedimientos aumentaron 12 veces su uso, además de un incremento del 18\% en el año de 1985 a un 95\% en el año 2005 en la toma de biopsias de esófago, estómago y duodeno. El 10\% de esta población tenia dolor abdominal recurrente en su mayoría no se encontró causa orgánica sin embargo a pesar de la evidencia estos pacientes a menudo eran referidos para endoscopia superior. $(9,10)$

El objetivo de este estudio fue determinar los hallazgos histológicos obtenidos por biopsias del tracto digestivo superior en el Servicio de Gastroenterología pediátrica del Hospital Mario Catarino Rivas en un período comprendido entre 1 de enero 2016 al 31 de mayo 2016, determinar características según edad y sexo de los pacientes sometidos a endoscopia digestiva superior, identificar el segmento anatómico del tracto digestivo superior del cual se obtuvo la muestra de biopsia, además establecer el diagnóstico clínico e histopatológico.

\section{MATERIALES Y METODOS}

Se realizó un estudio descriptivo transversal. El universo fueron todos los pacientes pediátricos a los que se realizó biopsia en el servicio de patología del HNMCR correspondiendo a una población de 224 en un período comprendido entre enero-mayo 2016, la muestra, 40 que cumplían con los criterios de inclusión: resultados de biopsia obtenidas por endoscopia superior en pacientes menores de 18 años; y los criterios de exclusión: pacientes mayores de 18 años y menores de 28 días, biopsias del tubo digestivo obtenidas mediante cirugía, biopsias aun sin reporte histopatológico, pacientes que llevaron biopsias a otro patólogo.

Las biopsias fueron realizadas en el servicio de endoscopia del HNMCR con el equipo Olympus Gift Q150 y Q160 en pacientes con indicación para dicho procedimiento y que cumplieron los criterios de inclusión para este estudio, las muestras para biopsia fueron obtenidas por medio de pinza Olympus $50 \mathrm{U} 1$ dirigidas a esófago, estómago y duodeno.

Las biopsias fueron enviadas al servicio de anatomía patológica en frascos con formol al $10 \%$ identificándose el segmento anatómico 
del cual fueron obtenidas, además se envió un informe de los hallazgos endoscópicos obtenidos, inicialmente se someten a la procesadora de tejido Leica TP 1020, se crearon bloques de parafina para posteriormente realizar el corte con micrótomo TBS cut4055; antes de realizar la tinción se desparafina en horno electrolux y se tiñieron con hematoxilina y eosina. Finalmente, son observados con el microscopio Oliympus CX21 y CX31 por patólogo de la institución. Los datos obtenidos fueron recabados de los libros y de los informes de biopsia del servicio de patología para su posterior análisis con medidas de frecuencia y porcentaje en el programa Microsoft Excel 2016.

\section{RESULTADOS}

Se encontró que de las 40 endoscopias realizadas, 19 (47.5\%) de los pacientes eran mayores de 11 años y 2 (5\%) eran preescolares.

(Ver tabla No. 1).

Tabla No. 1: Distribución de los pacientes según edad.

\begin{tabular}{ccc}
\hline Edad & $\mathbf{n}$ & Porcentaje \\
\hline $1-23 \mathrm{~m}$ & 6 & 15 \\
2-4 años & 2 & 5 \\
5-6 años & 9 & 22.5 \\
7-11 años & 4 & 10 \\
$>11$ años & 19 & 47.5 \\
Total & 40 & 100 \\
\hline
\end{tabular}

Fuente: Investigación, Hallazgos Histopatológicos, servicio anatomía patológica, HNMCR.

Del total de biopsias en estudio, 22 (55\%) fue en pacientes de sexo masculino y el 18 (45\%) del sexo femenino.

La toma de muestra mediante endoscopia de los pacientes en estudio fueron de esófago, estomago y duodeno; sin embargo el segmento anatómico especifico de la toma fue deacuerdo a los hallazgos macroscópicos observados, de esta forma se obtuvo la biopsia del antro del estomago 21 (38\%), seguida de unión gastroesofagica $15(27 \%)$, y 1 ra porción del duodeno 14 (25\%). (Ver tabla No. 2).

Tabla No. 2: Segmento anatómico de donde se obtuvo la muestra.

\begin{tabular}{lcc}
\hline \multicolumn{1}{c}{ Material enviado } & $\mathbf{n}$ & $\%$ \\
\hline Estómago (cuerpo) & 6 & 10 \\
Duodeno (1 ${ }^{\text {ra }}$ porción) & 14 & 25 \\
Esófago (unión gastoesofágica) & 15 & 27 \\
Estómago (antro) & 21 & 38 \\
Total & 56 & 100 \\
\hline
\end{tabular}

Fuente: Investigación, Hallazgos Histopatológicos, servicio anatomía patológica, HNMCR.

Las causas por las cuales se realizó endoscopia alta fue por dolor abdominal crónico en el 13 (33\%) de los pacientes seguido de enfermedad ácido péptica y reflujo gastroesofágico sin respuesta al tratamiento farmacológico 11 (28\%) y $8(20 \%)$ respectivamente.

(Ver tabla No. 3).

\section{Tabla No. 3: Diagnóstico clínico de los pacientes en estudio.}

\begin{tabular}{lcc}
\hline \multicolumn{1}{c}{ Diagnóstico clínico } & n & $\%$ \\
\hline Talla baja & 1 & 2 \\
Sangrado digestivo alto & 7 & 17
\end{tabular}

Reflujo gastroesofágico sin respuesta

$\begin{array}{lcc}\text { al tratamiento farmacologico } & 8 & 20 \\ \text { Enfermedad ácido péptica } & 11 & 28 \\ \text { Dolor abdominal crónico } & 13 & 33 \\ \text { Total } & 40 & 100\end{array}$

Fuente: Investigación, Hallazgos Histopatológicos, servicio anatomía patológica, HNMCR.

La gastritis crónica representó el hallazgo histopatológico más frecuente reportado en 24 (43\%) de los pacientes en estudio, ocupando el segundo lugar la esofagitis crónica con 15 (27\%) pacientes. (Ver tabla No. 4). 
Tabla No. 4: Diagnóstico histológico obtenido de biopsias del tracto digestivo.

\begin{tabular}{lcc}
\hline Diagnóstico patológico* & $\mathbf{n}$ & \% \\
\hline Antro congestivo & 1 & 2 \\
Duodeno normal & 3 & 5 \\
Duodenitis crónica & 4 & 7 \\
H. Pylori & 7 & 13 \\
Esofagitis crónica & 17 & 30 \\
Gastritis crónica & 24 & 43 \\
Total & 56 & 100 \\
\hline
\end{tabular}

*Se obtuvieron muestras de 2 o más segmentos anatómicos en distintos pacientes

Fuente: Investigación, Hallazgos Histopatológicos, servicio anatomía patológica, HNMCR.

Los adolescentes representaron el grupo etario con más hallazgos histopatológicos encontrándose principalmente gastritis, esofagitis crónica o ambas, vale la pena mencionar que el $85 \%$ de las pruebas positivas por $\mathrm{H}$. Pylori fueron pacientes adolescentes; los escolares y preescolares y adolescentes presentaron más de un hallazgo patológico por cada paciente. (Ver gráfico No. 1).

\section{Gráfico No. 1: Distribución por edades de los hallazgos histopatológicos.}

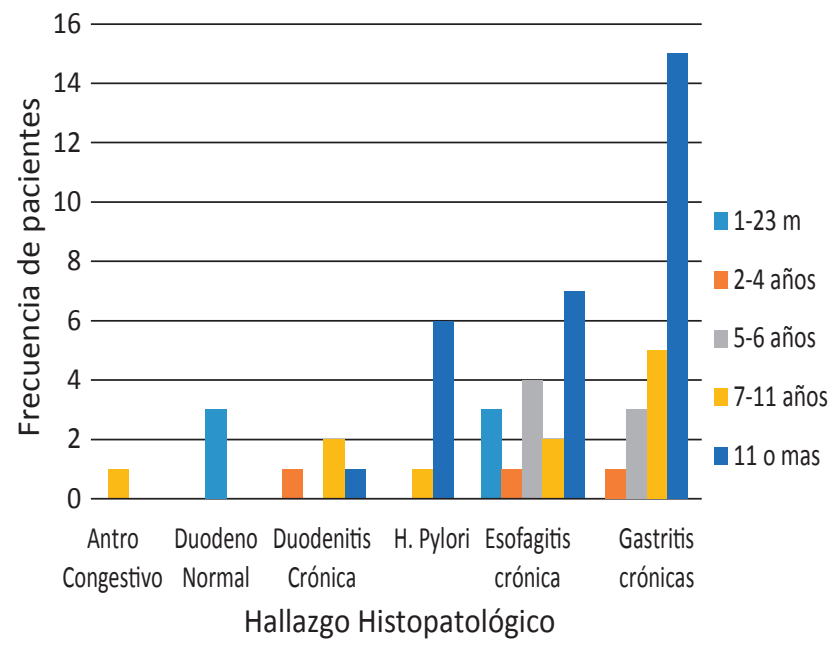

Fuente: Investigación, Hallazgos Histopatológicos, servicio anatomía patológica, HNMCR.

\section{DISCUSIÓN}

La endoscopia pediátrica es un procedimiento seguro independientemente de la edad ofrece tres ventajas: diagnóstica, seguimiento y terapéutica. Las enfermedades gastrointestinales tales como dolor abdominal crónico, vómitos y diarrea son comunes en alrededor de todo el mundo, muchas veces la endoscopia es el mejor método para el diagnóstico de la mayoría de estas enfermedades y su aplicación en los niños aumenta año con año. ${ }^{(3,11,12)}$

El costo de la endoscopia gastrointestinal en Honduras es alto y la mayoría de los pacientes son incapaces de pagar por estos servicios ofrecidos por el sector privado convirtiéndose el HNMCR en una opción para realizar dicho estudio.

Durante el período de investigación 19 (47.5\%) pacientes eran mayor de 11 años, un estudio realizado en Colombia encontró que la media de edad era de 15 años coincidiendo en que los adolescente son el grupo etario con mayor indicación de endoscopia. ${ }^{(13)}$

Otra publicación realizada por Sheiko et al, no se encontró diferencia significativa en cuanto al sexo puesto que de los 1000 pacientes en dicho estudio $49 \%$ fueron hombres, el estudio actual al igual que la literatura internacional no tuvo variabilidad considerable en cuanto relación hombre-mujer. ${ }^{(11,14,15)}$

Las indicaciones diagnósticas para realizar endoscopia según la Sociedad Europea de Gastroenterología, Hepatología y Nutrición (ESPGHAN) son: disfagia, odinofagia, síntomas intratables o crónicos de ERGE, vómitos/hematemesis, dolor epigástrico persistente, irritabilidad inexplicable, anorexia, pérdida de peso/ retraso en el desarrollo, anemia (inexplicable), diarrea/malabsorción (crónica), sangrado gastrointestinal, ingestión cáustica; y las indicaciones terapéuticas son: extracción de cuerpo extraño, dilatación de estenosis, ligadura de varices esofágicas, control de la hemo- 
rragia gastrointestinal superior; ${ }_{i}^{(16,17)}$ el presente estudio mostró que la principal indicación para realizar endoscopia fue dolor abdominal 13(33\%) y enfermedad acido péptica 11 (28\%), similar a estudios realizados en Estados Unidos y Colombia donde según datos obtenidos el dolor abdominal recurrente también fue la principal indicación de endoscopia en un $36 \%$ y $28 \%$ respectivamente, por otra parte indicaciones como reflujo gastroesofágico (11.7\%) y sangrado digestivo (19\%) ocuparon el segundo lugar en los países anteriormente mencionados; en otros países como Sudan donde la esquistosomiasis es endémica las principales indicaciones fueron hematemesis (24\%) e hipertensión portal (21\%), en Brazil la indicación más frecuente fue la sospecha de malabsorción y en segundo lugar el dolor abdominal recurrente. ${ }^{(11,16)}$

Dado que en la mayoría de los procesos patológicos del tubo digestivo la correlación endoscópica/histológica es muy baja es necesario realizar ambos estudios. En niños la discrepancia entre el aspecto endoscópico y el diagnóstico histológico puede ser de hasta más del $60 \%$ dependiendo de la técnica empleada, de la indicación y de la información clínica. La toma de las biopsias para estudio histológico o citológico, se efectúa una vez finalizada la exploración endoscópica. Se recomienda tomar muestras de esófago medio y distal. Dohil et al. y otros estudios refieren en su práctica que a nivel de estómago se deben tomar un mínimo de dos en la región prepilórica o antro y dos en la parte media del cuerpo ó curvatura mayor. ${ }^{(17)}$ Chairperson et al., recomiendan la toma de cuatro muestras a nivel de mucosa duodenal según la clínica del paciente. Kori et al. evaluaron la toma de biopsia duodenal de forma rutinaria durante la endoscopia digestiva superior en 201 pacientes pediátricos y al encontrar un valor predictivo negativo para el diagnóstico de mucosa normal de $81.5 \%$; concluyeron que la mucosa de apariencia macroscópica normal no descarta lesiones patológicas; sugirieron que la biopsia duodenal se debe hacer independientemente de la indicación de la endoscopia y de su apariencia macroscópica; por lo anteriormente expuesto se recomiendan la toma de muestras de varios segmentos anatómicos independientemente de que luzcan normales macroscópicamente, sin embargo en este estudio todas las biopsias iban dirigidas a esófago, estómago y duodeno, requiriendo dos o mas tomas de cada segmento en caso de encontrarse hallazgos anormales de la mucosa en la exploración endoscópica, como resultado los segmentos anatómicos mas afectados fueron antro 15 (38\%) y unión gastroesofágica $10(27 \%)$. $^{(11,13,18)}$

Según un estudio retrospectivo publicado por el hospital de niños de Colorado, de Estados Unidos donde se incluyeron 1000 pacientes, el $65 \%$ de los casos no hubo alteraciones endoscópicas y el $60 \%$ de los casos no mostro alteraciones histológicas en cualquiera de los sitios de biopsia enviadas al servicio de patología. Dhroove et al encontraron aún más el papel limitado para la endoscopia en cuanto a dolor abdominal se refiere, ya que solo el 9,7\% tenía hallazgos histopatológicos anormales, en contraste al estudio actual el hallazgo histopatológico encontrado en primer lugar fue gastritis crónica y en segundo lugar fue esofagitis crónica con 24 (43\%) y 17 (30\%) respectivamente, sin embargo llama la atención que solo 3 (5\%) de los pacientes no presentaron hallazgos histopatológicos lo que amerita una investigación adicional por el número alarmante de pacientes con resultados anormales; estas diferencias son probablemente explicadas por la práctica médica y la red en salud entre los Estados Unidos y los países en vías de desarrollo como el nuestro donde el estado nutricional y extrema pobreza juega un rol importante para la prevalencia de enfermedades gastrointestinales, agregado a esto podemos observar que se aisló $H$. Pylori en 7 (13\%) de los pacientes biopsiados de estos 6 (85\%) eran adolescentes; su prevalencia es baja en países desarrollados como Estados Unidos, describiéndose inferior al $5 \%$ en menores de 5 años, y de un $10 \%$ en 
adolescentes. Pero en países en vías de desarrollo aumenta a $50 \%$ en menores de 5 años y a un $90 \%$ en adultos. En el caso de Chile se ha descrito que los niños a los 4 años están colonizados en un $40 \%$ y los adolescentes en un $60 \% \cdot(7,19)$ La infección por este patógeno está relacionada con el nivel socioeconómico y las condiciones de vida del hospedero, a través de una vía directa (oral) específicamente el hacinamiento, la calidad del agua para el consumo doméstico, la falta de servicios higiénicos, la pertenencia a regiones con mayores niveles de ruralidad, ingresos familiares bajos y bajos niveles educacionales se han correlacionado históricamente con un aumento en la infección por H. pylori. ${ }^{(7,12,20)}$

En Conclusión, la endoscopia digestiva pediátrica ha evolucionado en las últimas décadas convirtiéndose en un procedimiento cada vez más frecuente, además es una herramienta diagnóstica segura y eficaz. Permite el seguimiento de enfermedades conocidas e identificación de trastornos emergentes. El apoyo del estudio histopatológico es de alta relevancia en nuestro medio considerando que el $95 \%$ de los pacientes presentaron hallazgos anormales en el resultado de la biopsia.

\section{BIBLIOGRAFÍA}

1. Giejournal.org (internet) American Society for Gastrointestinal Endoscopy 0016-5107 [actualizado 2014; consultado 8 abril 2016]. Disponible en: http://www.asge.org/uploa dedFiles/Publications_(public)/Practice_ guidelines/2014_Modifications\%20in\% 20endoscopic\%20practice\%20for\%20pedia tric\%20patients.pdf.

2. Thakkar Kalpesh, Gilger Mark A., Shulman Robert J., El Serag Hashem B. EGD in Children With Abdominal Pain: A Systematic Review. American Journal of Gastroenterology [revista en internet] 2007 [citado, 8 abril 2016] 102:654-661. doi: 10.1111/j.1572-02 41.2007.01051.x. disponible en: http:// www.nature.com/ajg/journal/v102/n3/ full/ajg2007117a.html.

3. Joo Kim Yong, General considerations and updates in pediatric gastrointestinal diagnostic endoscopy, Korean J Pediatr [revista en internet] 2010 [citado. 1 junio 2016] 53(9):817-823. DOI: 10.3345/kjp.2010.53.9. 817. disponible en: http://www.ncbi.nlm. nih.gov/pubmed/21189965.
4. Franciosi James P., Fiorino Kristin, Ruchelli Eduardo, Shults Justine, Spergel Jonathan, Liacouras Chris A., Leonard Mary, Changing Indications for Upper Endoscopy in Children During a 20-year Period, JPGN [revista en internet] 2010 [citado 1 junio 2016] 51(4):443-447. Disponible en: http://jour nals.lww.com/jpgn/pages/articleviewer. aspx year $=2010 \&$ issue $=10000 \&$ article $=$ 00011\&type.

5. Elitsur Yoram, The Diagnostic Yield of Upper Endoscopy Procedures in Children- Is it Cost Effective?, Curr Gastroenterol Rep [revista en internet] 2014 [citado 1 junio 2016] 16:385. DOI 10.1007/s11894-014-0 385-1. Disponible en: http://www.ncbi.nlm. nih.gov/pubmed/24676532.

6. Bishop Phyllis R., Nowick Michael J., May Warren L., Elkin David, Parker Paul H., Unsedated upper endoscopy in children. American Society for Gastrointestinal Endoscopy, [revista en internet] 2002 [citado, 8 abril 2016] 55(6):624-630. DOI: http://dx.doi.or g/10.1067/mge.2002.123417. Disponible 
en: http://www.giejournal.org/article/S001 6-5107(02)08056-2/abstract.

7. Mégraud Francis, Comparison of non-invasive tests to detect Helicobacter pylori infection in children and adolescents: Results of a multicenter European study, The Journal of pediatrics [revista en internet] 2005 [citado 8 abril 2016] 146(2):198-203. DOI: http://dx.doi.org/ 10.1016/j.jpeds.2004.10.044. Disponible en: http://www.jpeds.com/article/SOO 22-3476(04)00982-5/pdf.

8. Flóres Lucy S., Villalobos, Danielinne C. Rodríguez, Rosario López, Karolina C., et al, Endoscopia digestiva superior en pediatria, Colomb Med [revista en internet] 2005 [citado, 8 abril 2016] 36 (1): 42-51.

Disponible en: 36 (Supl 1): 42-51.

9. Gómez Zuleta Martín Alonso, Ruiz Morales Óscar Fernando, Riveros Javier, Utilidad de la endoscopia digestiva alta diagnóstica en pacientes menores de 18 años, Rev Col Gastroenterol [revista en internet] 2014 [ciatado 22 mayo 2016] 29 (2):111-115. Disponible en: http://www.scielo.org.co/ pdf/rcg/v29n2/en_v29n2a04.pdf.

10. Viada Bris J.F., Muñoz Codoceo R.A. , Endoscopia digestiva pediátrica, Pediatr Integral [revista en internet] 2015 [citado 8 abril 2016] 19(2):139-143. Disponible en: http:// www.pediatriaintegral.es/publicacion-20 15-02/endoscopia-digestiva-pedia trica.

11. Sheiko Melissa A., Feinstein James A., Capocelli Kelley E., Kramer Robert E., Diagnostic yield of EGD in children: a retrospective single-center study of 1000 cases, gastrointestinal endoscopy [revista en internet] 2013 [citado 8 abril 2016] 78(1):47-54. doi: http://dx.doi.org/10.1016/j.gie.2013. 03.168. Disponible en: http:// www. giejournal.org/article/S0016-5107(13)00 383-0/pdf.
12. Hernández Borjas Yanela, Banegas Ruth Maricela Caracterización Epidemiológica y Hallazgos Endoscópicos en pacientes menores de 14 años de edad con infección por Helicobacter pylori confirmado por biopsia en el Hospital Mario Rivas, de Septiembre 2009 a Mayo 2011, Acta Pediátrica Hondurena [revista en internet] 2011 [citado, 22 mayo 2016] 2(2):137-142. Disponible en: http:// www.bvs.hn/APH/pdf/APH Vol2/pdf/APHVol2-2-2011-2012-4.pdf.

13. Lee Way Seah, Zainuddin Hafizah, Boey Christopher CM, Fan Chai Pei, Appropriateness, endoscopic findings and contributive yield of pediatric gastrointestinal endoscopy, World J Gastroenterol [revista en internet] 2013 [citado, 22 mayo 2016], 19(47): 9077-9083. doi: 10.3748/wjg.v19.i47.9077. disponible en: http://www.ncbi.nlm.nih. gov/pmc/articles/PMC3870562.

14. Alurralde Carmen, Figueroa Frank, Soto Judith, Geraud Elisa, Mendoza Sonia, Folkmanas William, Tenia Juan, Linares Beatriz, Revisión de hallazgos histológicos de biopsias obtenidas por endoscópia digestiva superior de mayo 2006-2007, revista sociedad venezolana de gastroenterología [revista en internet] 2009 [citado, en 22 mayo 2016] 63(1):21-24. Disponible en: http://www.scielo.org.ve/pdf/gen/ v63n1/art05.pdf.

15. Gryboski, Joyce D. The value of upper gastrointestinal endoscopy in children, Digestive Diseases and Sciences [revista en internet] 1981 [citado 22 mayo 2016] 26 (7):17-21. Disponible en: http://link.sprin ger.com/article/10.1007\%2FBF01300801.

16. kostovski aco, Zdraveska nikolina, Indications for gastrointestinal endoscopy in children, Zdrav Vestn Supl [revista en internet] 2013 [citado 22 mayo 2016] 82(1):114-126. Disponible en: http://webca che.googleusercontent.com/search?q= 
cache:-0emMnXLul0J:www.dlib.si/ stream/ URN:NBN:SI:DOC-TAWQMXMB/3a03a97 f-2ea9-452f-bc05-2e9258a018e8/PDF+\&cd $=1 \& \mathrm{hl}=\mathrm{es} \& \mathrm{ct}=\mathrm{clnk} \& \mathrm{gl}=\mathrm{hn}$.

17. Ferreira Cristina T., Berti Mirela R., Pires Ana Luiza G., Wieczorek Cristiane, Alves Jorge, Endoscopia digestiva alta em pediatria: indicações e resultados, Jornal de Pediatria [revista en internet] 1998 [citado, 22 mayo 2016] 74(1):39-44. Disponible en: http:// www.jped.com.br/conteudo/98-74-01 $-39 /$ port.pdf.

18. Giejournal.org (internet) American Society for Gastrointestinal Endoscopy 0016-5107 [actualizado 2012, consultado 8 abril 2016]. Disponible en: http://www.giejournal.org/ article/S0016-5107(12)00188-5/pdf.
19. Soltani Jafar, Amirzadeh Jalil Nahedi Soheila, Shahsavari Sirous, Prevalence of Helicobacter Pylori Infection in Children, a Population-Based Cross-Sectional Study in West Iran, Iran J Pediatr. [revista en internet] 2013 [citado 22 mayo 2016], 23(1): 13-18.

Disponible en: http://www.ncbi.nlm.nih. gov/pmc/articles/PMC3574986/pdf/ IJPD-23-013.pdf.

20. De la Serna, C., Pérez-Miranda M., Fernández-Orcajo P., C. Alcalde, Caro-Patón A., retrospective study of pediatric endoscopy as performed in an adult endoscopy unit, REV ESP ENFERM DIG [revista en internet] 2010 [citado 22 mayo 2016] 102 (2): 100-107. Disponible en: http://www.ncbi. nlm.nih.gov/pubmed/20361846. 